hep-th/9609070, DUKE-TH-96-130, RU-96-80

\title{
Extremal Transitions and Five-Dimensional Supersymmetric Field Theories
}

\author{
David R. Morrison \\ Department of Mathematics, Box 90320 \\ Duke University \\ Durham, NC 27708-0320 USA \\ drm@math.duke.edu \\ and \\ Nathan Seiberg \\ Department of Physics and Astronomy \\ Rutgers University \\ Piscataway, NJ 08855-0849 \\ seiberg@physics.rutgers.edu
}

We study five-dimensional supersymmetric field theories with one-dimensional Coulomb branch. We extend a previous analysis which led to non-trivial fixed points with $E_{n}$ $\operatorname{symmetry}\left(E_{8}, E_{7}, E_{6}, E_{5}=\operatorname{Spin}(10), E_{4}=S U(5), E_{3}=S U(3) \times S U(2), E_{2}=S U(2) \times\right.$ $U(1)$ and $\left.E_{1}=S U(2)\right)$ by finding two new theories: $\widetilde{E}_{1}$ with $U(1)$ symmetry and $E_{0}$ with no symmetry. The latter is a non-trivial theory with no relevant operators preserving the super-Poincaré symmetry. In terms of string theory these new field theories enable us to describe compactifications of the type I' theory on $\mathbf{S}^{\mathbf{1}} / \mathbb{Z}_{\mathbf{2}}$ with 16,17 or 18 background D8branes. These theories also play a crucial role in compactifications of M-theory on CalabiYau spaces, providing physical models for the contractions of del Pezzo surfaces to points (thereby completing the classification of singularities which can occur at codimension one in Kähler moduli). The structure of the Higgs branch yields a prediction which unifies the known mathematical facts about del Pezzo transitions in a quite remarkable way.

September, 1996 


\section{Introduction}

Local quantum field theories occur in string theory in two places. They describe both the low energy excitations in string compactifications and those on D-branes [1]. Therefore, we have three distinct parallel lines of research: local quantum field theory, the theory of D-branes, and string compactification. It is extremely interesting to understand the relation between these three topics and to establish a complete dictionary translating concepts from one topic to the others.

The traditional approach has been to use field theory results to learn about properties of string theory. Recently, however, the opposite logic turned out to be fruitful. The assumption of string duality was used to shed new light on interesting field theories [2, 3]. In particular, in [3] the five-dimensional field theory on a D4-brane probe was studied. The analysis led to the discovery of new non-trivial fixed points. As we said above, these theories also appear in string compactifications. Here we complete the picture by relating this new field theoretic understanding to the geometry of the compactification.

In section 2 we will review the analysis of [3] and will extend it. We will consider $U(1)$ and $S U(2)$ field theories with $N_{f}$ quark flavors and will describe their low energy field theory. We will show how non-trivial fixed points with $E_{n}$ symmetry $\left(E_{8}, E_{7}, E_{6}\right.$, $E_{5}=\operatorname{Spin}(10), E_{4}=S U(5), E_{3}=S U(3) \times S U(2), E_{2}=S U(2) \times U(1)$ and $\left.E_{1}=S U(2)\right)$ can be found. We will also show that upon suitable perturbations these theories can flow to two new theories: $\widetilde{E}_{1}$ with $U(1)$ symmetry and $E_{0}$ with no symmetry.

These results are derived using the assumption of type I/type I'/heterotic duality. They enable us to extend the type I' description to the entire moduli space of vacua including vacua where at some point in space-time the coupling constant diverges [4, 四]. We find that while in type $\mathrm{I}^{\prime}$ compactifications on $\mathbf{S}^{\mathbf{1}} / \mathbb{Z}_{\mathbf{2}}$ at weak coupling there are two orientifolds and 16 D8-branes, at strong coupling there can be 17 or 18 D8-branes. This can happen only when the string coupling at one or at both ends of $\mathbf{S}^{\mathbf{1}} / \mathbb{Z}_{\mathbf{2}}$ diverges.

The other application of these field theories is discussed in section 3. Five-dimensional field theories occur in compactifications of M-theory on a Calabi-Yau threefold. Such compactifications were studied in [5 8]. Of particular interest are the compactifications on singular Calabi-Yau spaces. Following Strominger [9], we should understand these singularities in terms of new degrees of freedom which become massless at that point. Such degrees of freedom can enable one to understand transitions to different compactifications [10]. Witten has performed such an analysis in [7] for two kinds of singularities. As in four dimensions, the conifold singularity [9,10] was interpreted as an Abelian gauge theory

with new massless hypermultiplets and the extremal transition of [11,12] was interpreted as an enhanced non-Abelian gauge theory. 
Here, we complete the story by identifying the physics of the third and final type of singularity whose geometry is that of a four-manifold within the Calabi-Yau (a so-called "del Pezzo surface") which shrinks to zero size at the transition point. These turn out to be the interacting supersymmetric five-dimensional theories mentioned above.1 $\mathrm{In}$ such interacting conformal field theories the notion of a particle is ill defined. Therefore, it is meaningless to ask which particles are massless at that point in the moduli space.

The known mathematical classification of del Pezzo surfaces precisely matches the list of interacting field theories we have found. Furthermore, the identification of the symmetry group at the transition point as one of the $E_{n}$ groups yields the prediction that the Higgs branch of these theories will coincide with the corresponding instanton moduli space. That prediction unifies the known mathematical facts about these del Pezzo transitions in a quite remarkable way, and suggests a uniform structure which had not previously been suspected.

\section{Five-dimensional supersymmetric field theories}

In this section we review and extend the discussion in [3] of five-dimensional supersymmetric field theories. The massless fields are in hypermultiplets or vector multiplets. These theories typically have a moduli space of vacua. The scalars in the hypermultiplets parameterize a hyper-Kähler manifold which we will refer to as the Higgs branch. The scalars in the vector multiplets $\mathcal{A}^{i}$ parameterize the Coulomb branch. The metric on the Coulomb branch is determined by a prepotential $\mathcal{F}\left(\mathcal{A}^{i}\right)$ which is locally cubic

$$
(d s)^{2}=\frac{\partial^{2} \mathcal{F}}{\partial \mathcal{A}^{i} \partial \mathcal{A}^{j}} d \mathcal{A}^{i} d \mathcal{A}^{j} .
$$

The metric determines the kinetic terms of the scalars in the multiplets and the gauge coupling constants for the "photons" in the vector multiplets. Since $\mathcal{F}$ is cubic, the metric varies linearly. The singularities in the Coulomb branch are of two kinds. First, the moduli space of vacua could have boundaries. Second, the space could be smooth but the metric (2.1) could be non-differentiable. At such points the slope of the metric changes.

As in [3], we limit ourselves to theories with a one-dimensional Coulomb branch parameterized by a real scalar $\phi$. The metric and the gauge coupling are determined by a piecewise linear function

$$
t(\phi)=\frac{1}{g^{2}(\phi)}=t_{0}+c \phi
$$

1 The observation that the $E_{n}$ theories of [3] explain the physics of del Pezzo contractions was independently made by Vafa [13], and is being further developed by Douglas, Katz, and Vafa [14]. 
Singularities of the first kind correspond to a Coulomb branch being $\mathbf{R}^{+}$. The gauge coupling near the origin is of the form (2.2). If $c>0$, we could also have $t_{0}=0$. Singularities of the second kind have a Coulomb branch $\mathbf{R}$. Around the singular point $\phi_{0}$ the metric is determined by

$$
t(\phi)=t_{0}+c_{0} \phi+c\left|\phi-\phi_{0}\right|
$$

The critical behavior (the singularity) is again characterized by the value of $c$.

The simplest theories studied in [3], are $U(1)$ gauge theories with $N_{f}$ "electrons." At tree level $t=t_{0}$ is independent of $\phi$. However, a one-loop correction [7] induces non-zero $c$ such that

$$
t(\phi)=t_{0}-\sum_{i=1}^{N_{f}}\left|\phi-m_{i}\right|
$$

where $m_{i}$ are the electron masses. We would like to make a few comments on (2.4)

(1.) By shifting $\phi$ we can change $m_{i}$ such that only $N_{f}-1$ of them are important.

(2.) The one-loop computation is divergent. Therefore, we had to absorb an additive (non-universal) divergent renormalization in $t_{0}$.

(3.) There could also be a finite correction to $t_{0}$. On dimensional grounds, any such correction must be linear in the masses $m_{i}$. Using the symmetries, it must also be proportional to $\sum_{i} m_{i}$. We can absorb such a potential contribution in $t_{0}$.

(4.) The expression (2.4) makes sense only when $t(\phi)$ is non-negative. This means that $t_{0}$ should be positive and the $m_{i}$ are constrained. Furthermore, for any $t_{0}$ and $m_{i}, \phi$ is bounded. In other words, the moduli space always has singularities. These reflect the lack of renormalizability of the theory, signaling that it must be embedded in another theory at short distance.

The gauge coupling $g=\frac{1}{\sqrt{t}}$ has negative mass dimension and therefore it is "irrelevant" at long distance. Hence, the degrees of freedom at the singularities are the fundamental photon and electrons.

When several masses are equal, several singularities in (2.4) coalesce. Consider the case where all the masses are equal. Shifting $\phi$ we can set the mass to zero. The global symmetry of the theory is then $S U\left(N_{f}\right)=A_{N_{f}-1}$ (it is not $U\left(N_{f}\right)$ because the $U(1)$ factor is gauged). Equation (2.4) becomes

$$
t(\phi)=t_{0}-N_{f}|\phi|
$$

The degrees of freedom at the singularity are again the elementary photon and electrons. There is a Higgs branch emanating from the origin. It is isomorphic to the moduli space of $S U\left(N_{f}\right)$ instantons. 
We will refer to these theories as $A_{N_{f}-1}$ theories reflecting their global symmetry and their Higgs branch. We will extend this terminology to the case of $N_{f}=1$ and will refer to this latter theory as $A_{0}$.

The second class of theories consists of $S U(2)$ gauge theories with $N_{f}$ "quarks" which are hypermultiplets in the two-dimensional representation of the gauge group. Now the Coulomb branch is $\mathbf{R}^{+}$parameterized by $\phi \geq 0$. The one-loop computation yields

$$
t(\phi)=t_{0}+16 \phi-\sum_{i=1}^{N_{f}}\left|\phi-m_{i}\right|-\sum_{i=1}^{N_{f}}\left|\phi+m_{i}\right| .
$$

We will make similar comments to the ones above, but with some crucial differences:

(1.) We cannot shift $\phi$ and therefore all the masses are physical.

(2.) The one-loop computation is again divergent and we absorbed an additive (nonuniversal) divergent renormalization in $t_{0}$.

(3.) There could again be finite corrections to $t_{0}$. On dimensional grounds they have to be linear in the masses $m_{i}$. For $N_{f} \geq 2$ the global $S O\left(2 N_{f}\right)$ symmetry of the theory with $m_{i}=0$ prevents such corrections.

(4.) The expression (2.6) makes sense only when $t(\phi)$ is non-negative. This means that $t_{0}$ should be non-negative and $m_{i}$ are constrained. For $N_{f}>8 \phi$ is bounded. As in the $U(1)$ theories this reflects the lack of renormalizability of the theory signaling that it must be embedded in another theory at short distance.

(5.) For $N_{f}=8$ and $t_{0}>2 \sum\left|m_{i}\right|$ there is no singularity in the moduli space. If we try to set all $m_{i}=0$ and then take the strong coupling limit $t_{0} \rightarrow 0$, the metric on the entire moduli space vanishes and hence the limit is meaningless.

(6.) The theories with $N_{f} \leq 7$ are very interesting and will be discussed below.

The physical particles at the singularities of (2.6) with non-zero $\phi$ consist of one of the elementary gauge bosons and one of the elementary quarks. The low energy theory is thus the $A_{0}$ theory discussed above. (Note that the singularity in $t$ is exactly as in that theory). If $k$ masses are equal but do not vanish, there is a singularity with $k$ massless charged particles. The theory at that singularity is the $A_{k-1}$ theory,

New theories are found around $\phi=0$. If $N_{f}$ quark masses vanish, the global symmetry is $S O\left(2 N_{f}\right)$ and we will refer to the theory as $D_{N_{f}}$. There is also another interesting $U(1)$ symmetry whose conserved charge is the instanton number and whose conserved current is $j={ }^{*}(F \wedge F)$. It does not act on the massless particles. Here, the massless particles at the singularity are the three gauge bosons and $N_{f}$ massless quark hypermultiplets. Also, there is a Higgs branch isomorphic to the moduli space of $S O\left(2 N_{f}\right)$ instantons emanating from that point. 
For $N_{f} \leq 7$ we can consider the strong coupling limit $t_{0} \rightarrow 0$. The main point of [3] was that this limit exists. The theory at the singularity at $\phi=0$ is a non-trivial interacting fixed point of the renormalization group. The notion of a particle at this point is ill defined. For $m_{i}=0$ these theories haves $E_{N_{f}+1}$ global symmetry with $E_{5}=S p i n(10), E_{4}=S U(5)$, $E_{3}=S U(3) \times S U(2), E_{2}=S U(2) \times U(1)$ and $E_{1}=S U(2)$. Clearly, their Coulomb branch is $\mathbf{R}^{+}$. The function $t$ is obtained from (2.6)

$$
t(\phi)=\left(16-2 N_{f}\right) \phi
$$

Less obvious is the fact that their Higgs branch is isomorphic to the moduli space of $E_{N_{f}+1}$ instantons. (For $N_{f}=2$ there are two separate Higgs branches - along one of them $S U(3)$ is broken and along the other $S U(2)$ is broken).

It is convenient to think of the parameters in these theories as background superfields [15]. In these theories they are scalars in background vector superfields [16] associated with the global symmetry of the theory. This interpretation makes it obvious that they have dimension one. The D-term equations force them to lie in the Cartan subalgebra. Therefore, their number is the rank of the algebra. Indeed, our $A_{N_{f}-1}$ theory has $N_{f}-1$ parameters $\left(N_{f}\right.$ masses, one linear combination of which can be removed by redefining $\left.\phi\right)$, and the $D_{N_{f}}$ theory has $N_{f}$ parameters (the $N_{f}$ masses) while deformations of the gauge coupling $t_{0}$ from its non-zero value is an irrelevant parameter. In the $E_{N_{f}+1}$ theory there are $N_{f}+1$ parameters, namely, $m_{i}$ and $t_{0}$.

Since the parameters $t_{0}$ and $m_{i}$ have dimension one, the corresponding operators are relevant at the fixed points. Turning on $t_{0}$ with $m_{i}=0$ takes us to the $D_{N_{f}}$ theory. Flows between these theories are obtained by turning on some of the $m_{i}$. To keep $t=0$ at $\phi=0$ we should tune $t_{0}$ to $2 \sum_{i}\left|m_{i}\right|$. Then, the parameter " $t_{0}$ " of the low energy theory is $t_{0}-2 \sum_{i}\left|m_{i}\right|$.

Let us consider the case $N_{f}=1$ in more detail. As we said above, for $N_{f} \geq 2$ the global $S O\left(2 N_{f}\right)$ helped us identify $t_{0}$ as an $S O\left(2 N_{f}\right)$ singlet with no finite additive mass renormalization in (2.6). For $N_{f}=1$ the global symmetry is $E_{2}=S U(2) \times U(1)$. The single mass $m_{1}=m$ is associated with the $U(1)$ factor. We would like to identify the parameter $m_{0}$ associated with the $S U(2)$ breaking. The singularities of the gauge coupling occur at $t_{0}=2|m|$. Since there is no symmetry under which $m$ changes its sign 2 , the natural variable $m_{0}$ can be a linear combination of $t_{0}$ and $m$. Without loss of generality we define

$$
m_{0}=t_{0}-2 m
$$

2 The $D_{N_{f}}$ theories have $S O\left(2 N_{f}\right)$ but not $O\left(2 N_{f}\right)$ symmetry. 
such that

$$
t(\phi)=m_{0}+2 m+16 \phi-|\phi-m|-|\phi+m| .
$$

This expression is valid only for $m_{0}+4 m>0$. Since $m_{0}$ is associated with the $S U(2)$ factor, the Weyl subgroup identifies $m_{0}$ with $-m_{0}$. To keep the equations simple we will restrict $m_{0}$ to be non-negative (as with $\phi$ ).

For $m_{0}=m=0$ the theory is our $E_{2}$ theory. Turning on $m_{0}$ with $m=0$ leads to a flow to the trivial $D_{1}$ theory. If we instead turn on $m>0$ but keep $m_{0}=0$, we flow to the $E_{1}$ theory with $E_{1}=S U(2)$ global symmetry (see figure 1 ). At $\phi=m$ we find an $A_{0}$ theory. For non-zero $m_{0}$ and $m>0$ we flow to the trivial $D_{0}$ theory. Again, at $\phi=m$ we find an $A_{0}$ theory.

Since there is no symmetry changing the sign of $m$, we do not expect similar results for $m<0$. In particular, there is a singularity at $m_{0}+4 m=0$ without a counterpart for positive $m$. For $m_{0}+4 m>0$ and $m<0$ the perturbative analysis is still valid and the low energy theory at $\phi=0$ is $D_{0}$ and at $\phi=-m$ it is $A_{0}$. The lack of $m \rightarrow-m$ symmetry appears only in the massive modes. Therefore, we will distinguish this theory from $D_{0}$ by denoting it $\widetilde{D}_{0}$. The distinction between them becomes important only when we approach $m_{0}+4 m=0$. At this point the gauge coupling diverges $t=0$. However, the transition to this point is different from the transition from $D_{0}$ to $E_{1}$. The $E_{1}$ theory has $S U(2)$ global symmetry while the theory at $m_{0}+4 m=0$ has only $U(1)$ global symmetry. Therefore, this theory must be a new non-trivial fixed point in five dimensions. We will refer to it as $\widetilde{E}_{1}$. Clearly it has only one relevant parameter $s=m_{0}+4 m$. Using arguments similar to those in [3], we learn that there is no Higgs branch emanating from it.

We now explore the region $m_{0}+4 m<0$ (as before with $m_{0}$ non-negative). We do that by turning on the only relevant parameter in $\widetilde{E}_{1}$, namely, $s=m_{0}+4 m$. For $s>0$ we flow to $\widetilde{D}_{0}$. What happens for $s<0$ ? We propose that the transition is similar to other transitions we saw before where an $A_{0}$ theory leaves the origin. The theory at the origin is then at a new non-trivial fixed point with $t=0$ at $\phi=0$ which we will call $E_{0}$. Before arguing that such a theory indeed exists, let us use this proposal to describe the physics for $s<0$. The global $U(1)$ symmetry of the $\widetilde{E}_{1}$ theory acts on the particle which leaves the origin at the transition. Therefore, there is no symmetry acting on the massless modes in $E_{0}$. This fact is consistent with the lack of relevant parameters in this theory.

The behavior near $\phi=0$ discussed above does not affect the $A_{0}$ theory near $\phi=-m$. This should remain unaffected even for $m_{0}+4 m<0$ and in particular at $m_{0}=0$ where the global $S U(2)$ symmetry must be restored. Now, let us track the $A_{0}$ theory which leaves the origin. It corresponds to a $U(1)$ gauge theory with one electron with mass $($ at $\phi=0)$ $-\alpha s$ ( $\alpha$ is a positive proportionality factor which we will determine momentarily) such 


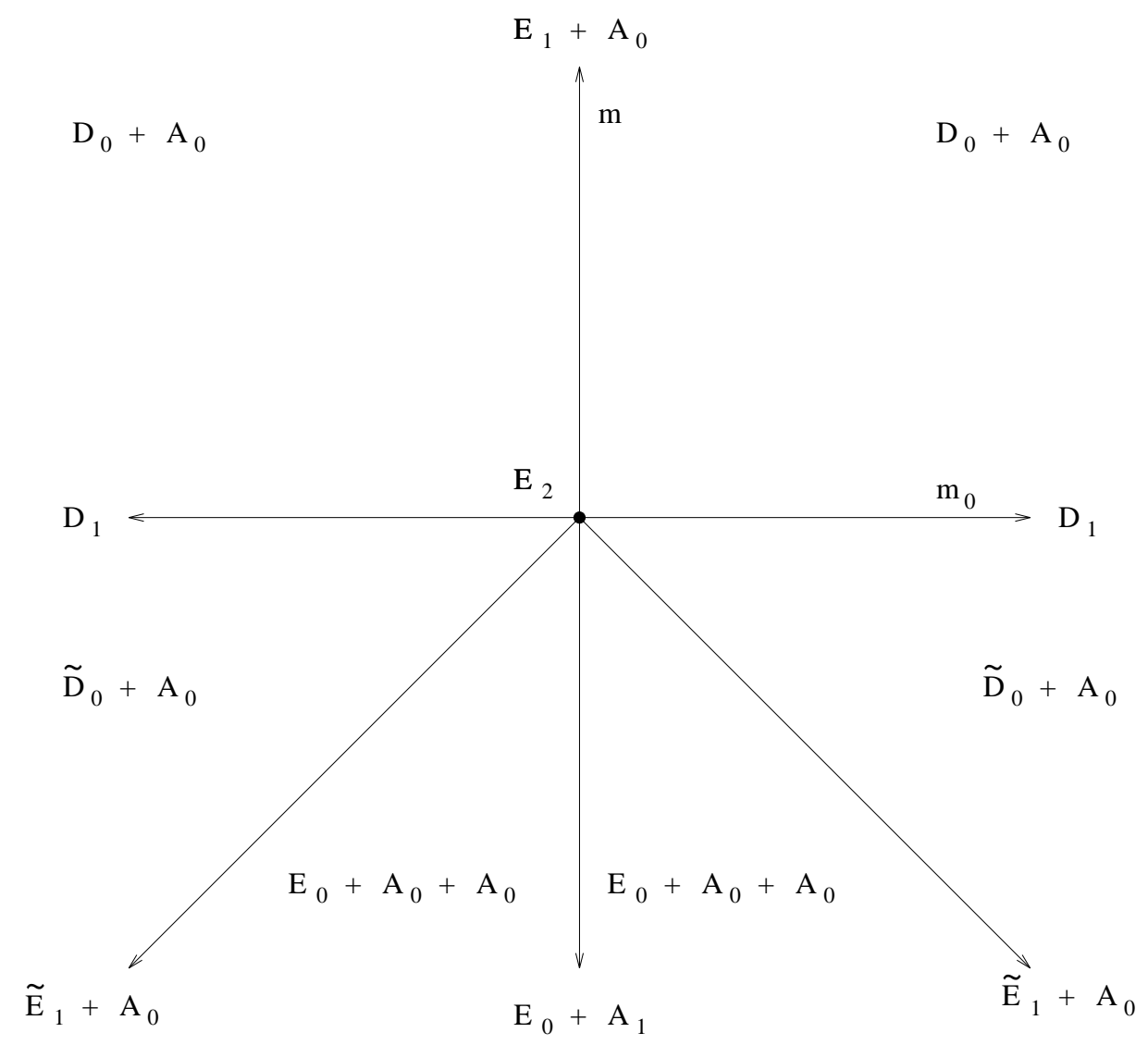

Figure 1. $E_{2}$ and related theories.

that the singularity is at $\phi=-\alpha s$. As we make $s$ more negative (say with fixed $m$ ), this singularity approaches the singularity at $\phi=-m$. For $m_{0}=0$ we must regain the global $S U(2)$ symmetry. The only way this could happen out of our setup with $E_{0}$ at the origin without symmetries and the two $A_{0}$ singularities is if the $A_{0}$ 's coalesce to an $A_{1}$ theory (see figure 1). This fixes $\alpha=\frac{1}{4}$.

Assuming that $t=0$ at $\phi=0$ in the region with $E_{0}$ (i.e., that the origin of $\phi$ does not move), we can find a simple formula for the function $t$ which is valid for all $\phi \geq 0$ and $m_{0} \geq 0$ :

$$
t(\phi)=\frac{m_{0}}{2}+3\left|m+\frac{m_{0}}{4}\right|+17 \phi-|\phi+m|-|\phi-m|-\left|\phi+m+\frac{m_{0}}{4}\right| .
$$

It exhibits all the singularities we discussed above.

This phenomenon has a generalization to higher $E_{n}$. The $t_{0}$ perturbation breaks $E_{n}$ to $S O(2 n-2) \times U(1)$. Turning on also equal masses to all the quarks $m_{i}=m$ breaks the symmetry to $S U(n-1) \times U(1) \times U(1)$ with an $A_{n-2}$ singularity away from the origin and 
a $D_{0}$ singularity at the origin. There is another way to get to this point. First, we can break $E_{n}$ to $S U(n) \times U(1)$ by choosing suitable values for $t_{0}$ and $m_{i}$. We argue that in this case we have an $E_{0}$ theory at the origin and an $A_{n-1}$ theory away from the origin. As the symmetry is further broken to $S U(n-1) \times U(1) \times U(1)$ the $A_{n-1}$ singularity splits to an $A_{n-2}$ and an $A_{0}$ singularity. This can move to the origin, be absorbed in it to turn it into an $\widetilde{E}_{1}$ theory which can then become $\widetilde{D}_{0}$, which can then become $D_{0}$ exactly as in the other way of getting there.

As in [3], all this has a simple translation to the physics of branes in string compactification. We use a D4-brane probe in the compactification of the type $\mathrm{I}^{\prime}$ theory on $\mathbf{S}^{\mathbf{1}} / \mathbb{Z}_{\mathbf{2}}$. This theory is dual to the heterotic string compactified on $\mathbf{S}^{\mathbf{1}}$. The $A_{n}$ theories are obtained when $n+1$ background D8-branes coalesce in the interior of $\mathbf{S}^{\mathbf{1}} / \mathbb{Z}_{\mathbf{2}}$. The $D_{n}$ theories are obtained when $n$ D8-branes are at an orientifold at the boundary of $\mathbf{S}^{\mathbf{1}} / \mathbb{Z}_{\mathbf{2}}$. The $E_{n}$ theories are obtained when the string coupling diverges on the boundary. The existence of the new $E_{0}$ theory implies that we can have more than 16 background D8-branes in the interior. In particular, if the theory at one of the boundaries is at $E_{0}$, we can have 17 background D8-branes and if the two boundaries are at $E_{0}$, we can have 18 background D8-branes. If these 18 D8-branes coalesce, the symmetry group of the string theory is $S U(18) \times U(1)$. In terms of the dual heterotic string the $S U(18)$ factor comes from the left movers and the $U(1)$ from the right movers.3 Note that although the string coupling diverges at the two boundaries, it can be arbitrarily weak at the point in the middle of $\mathbf{S}^{\mathbf{1}} / \mathbb{Z}_{\mathbf{2}}$ where the background D8-branes are. Similarly, we could have one boundary with $E_{0}$ and 17 background D8-branes near an orientifold at the other boundary yielding an $S O(34) \times U(1)$ symmetry. Again, the physics near the orientifold where the $S O(34)$ gauge bosons are can be arbitrarily weakly coupled.

Finally, we argue for the existence of the $E_{0}$ theory. The presence of $S U(18) \times U(1)$ and $S O(34) \times U(1)$ points in the moduli space of the heterotic string and the symmetrybreaking pattern tell us that the type $\mathrm{I}^{\prime}$ theory must have corresponding points where 18 D8-branes coincide, or 17 D8-branes are at an orientifold. This also lets us reproduce the transitions to the $\widetilde{E}_{1}$ and $\widetilde{D}_{0}$ theories, guaranteeing that our scenario for the phase diagram and the existence of the $E_{0}$ point is correct.

3 Denote by $\mathbf{n}$ the representation of $S U(18)$ Kac-Moody level one whose highest weight vector is in a representation of $S U(18)$ constructed as an antisymmetric product of $n$ fundamentals. Denote its character by $\chi_{\mathbf{n}}(q)$. Then, the partition function $\left|\chi_{\mathbf{0}}+\chi_{\mathbf{6}}+\chi_{\mathbf{1 2}}\right|^{2}+\left|\chi_{\mathbf{3}}+\chi_{\mathbf{9}}+\chi_{\mathbf{1 5}}\right|^{2}$ is an exceptional modular invariant of a consistent conformal field theory. Denote by $\psi_{\mathbf{0}}$ and $\psi_{\mathbf{1}}$ the characters of the two representations of $S U(2)$ Kac-Moody level one. Then the partition function $Z=\left(\chi_{\mathbf{0}}+\chi_{\mathbf{6}}+\chi_{\mathbf{1 2}}\right) \overline{\psi_{\mathbf{0}}}+\left(\chi_{\mathbf{3}}+\chi_{\mathbf{9}}+\chi_{\mathbf{1 5}}\right) \bar{\psi}_{\mathbf{1}}$ is modular invariant. This is the partition function of the conformal field theory based on the $(17,1)$ signature lattice for this $S U(18)$ theory. 


\section{Extremal transitions in the geometry}

In the previous section, we have described supersymmetric five-dimensional field theories with a one-dimensional Coulomb branch, and given the D4-brane interpretation of these theories. We now turn to the other string-theoretic application of the field theory analysis: M-theory compactified on a Calabi-Yau threefold.

In such M-theory compactifications, the expectation values for the scalars in vector multiplets are parameterized by the Kähler classes of volume one on the Calabi-Yau threefold [5,60,8]. The Kähler cone (i.e., the set of all Kähler classes) has various boundary "walls," and we should expect interesting physics as we approach such a wall since the metric on the Calabi-Yau threefold is degenerating there. Walls along which $\int J \wedge J \wedge J$ vanishes will be at infinite distance from the interior of the moduli space, since we must divide a Kähler class $J$ by $\sqrt[3]{\int J \wedge J \wedge J}$ in order to get a class of volume one. along which $\int J \wedge J \wedge J$ does not vanish corresponds to the collapse of some proper subset of the Calabi-Yau threefold [18], for if the entire Calabi-Yau collapses, then $\int J \wedge J \wedge J$ must vanish.

The possible ways that a proper subset of a Calabi-Yau threefold can collapse upon approaching a boundary wall of the Kähler cone have been analyzed in considerable detail in the mathematics literature. We restrict our attention to codimension-one walls, so that approaching the wall involves tuning only a single vector multiplet expectation value; we also assume that the complex structure on the Calabi-Yau threefold is generic (so that no hypermultiplets need to be tuned to reach the singularity). Under these assumptions, the possible types of "collapse" are the following:

(1.) A collection of $N$ disjoint $\mathbb{P}^{1}$ 's can collapse to $N$ "ordinary double points."

(2.) A rational surface which has a "birational ruling," $N$ fibers of which consist of a pair of $\mathbb{P}^{1}$ 's and the remaining fibers of which are irreducible $\mathbb{P}^{1}$ 's, can collapse along the ruling to a $\mathbb{P}^{1}$ of singularities.

(3.) A "del Pezzo surface" 6 which is the blowup of $\mathbb{P}^{1} \times \mathbb{P}^{1}$ at $N$ points $(0 \leq N \leq 7)$, or of $\mathbb{P}^{2}$ at $N+1$ points $(0 \leq N+1 \leq 8)$, can collapse to a point. (When $N \geq 1$, the two descriptions are equivalent.)

4 This is the analogue in five dimensions of Hayakawa's criterion [17] in four dimensions, which specifies which singularities are at finite distance in the Zamolodchikov metric.

5 When the complex structure on the Calabi-Yau threefold is not generic, there are further types of collapse which can occur, described in the appendix. We should point out here that the possibility is left open by the literature that one or two additional cases might need to be included along with the ones we present.

6 A del Pezzo surface is a complex manifold $S$ of complex dimension 2 whose anticanonical divisor $-K_{S}$ is ample, i.e., $-K_{S} \cdot C>0$ for every Riemann surface $C$ contained in $S$. (See [19,20], 
Anticipating our field theory identifications, we shall refer to these three cases as $A_{N-1}$, $D_{N}$, and $E_{N+1}$ (or $\widetilde{E}_{1}$ ) respectively (where $E_{1}$ is the case of $\mathbb{P}^{1} \times \mathbb{P}^{1}$ blown up at 0 points, $\widetilde{E}_{1}$ is the case of $\mathbb{P}^{2}$ blown up at 1 point, and $E_{0}$ is the case of $\mathbb{P}^{2}$ blown up at 0 points).

Although the "collapse" in each of these cases is the result of a degeneration of the metric on the Calabi-Yau, the resulting collapsed space can be described using algebraic geometry as the solution set of a collection of polynomial equations. (The algebraic versions of such collapses are known as "extremal contractions" [22], and have been studied extensively in the mathematics literature.) Since the singularities have an algebraic description, algebraic methods can be used to determine whether or not the collapsed space can be smoothed out by varying the coefficients in the polynomial equations. If this is possible, the smoothed space is again a Calabi-Yau threefold, but of a completely different topology than the original one. The process of collapsing via an extremal contraction and then smoothing out the resulting space to produce a new Calabi-Yau threefold is known as an "extremal transition" [23].

It will be useful to consider a slight generalization of this setup, in which the geometry of the contraction is similar but the contraction may occur at higher codimension in the Kähler cone. Let $S$ be either (1) a collection of $\mathbb{P}^{1}$ 's, (2) a birationally ruled surface over $\mathbb{P}^{1}$, or (3) a del Pezzo surface, and suppose that $S$ is embedded in a Calabi-Yau threefold $X$ in such a way that there is a contraction $X \rightarrow \bar{X}$ associated to some wall of the Kähler cone of $X$ which maps $S$ to (1) ordinary double points, (2) a $\mathbb{P}^{1}$ of singularities, or (3) a singular point, respectively. If the codimension of the wall of the Kähler cone is $k$, then the dimension of the image of the natural map $H^{1,1}(X) \rightarrow H^{1,1}(S)$ is $k+\epsilon$, where $\epsilon=0$ in cases (1) and (3), and $\epsilon=1$ in case (2).

In the $A_{N-1}$ case (in this more general sense), a physical interpretation of the contraction and extremal transition was found in [9, 10] when the type IIA string is compactified on the Calabi-Yau threefold: 7 there are $k U(1)$ 's associated to the homology classes spanned by the $\mathbb{P}^{1}$ 's, there are $N$ massive hypermultiplets charged under $U(1)^{k}$ which become simultaneously massless at the transition point, and there are $N-k$ flat directions at the transition which produce $N-k$ new parameters (the Higgs branch in the field theory). Mathematically, it was already known that this extremal transition exists [26], and that

or for a brief account in the physics literature, [21].) Every such surface is known to be either $\mathbb{P}^{1} \times \mathbb{P}^{1}$, or a blowup of $\mathbb{P}^{2}$; when more than one point of $\mathbb{P}^{2}$ has been blown up the surface can also be described as a blowup of $\mathbb{P}^{1} \times \mathbb{P}^{1}$.

7 The analysis of [9,10] was done in type IIB language, but as has been pointed out in [24,25], the same analysis can be applied to the type IIA string. 
the number of new complex deformation parameters would be $N-k$ [27,28]. (These parameters, together with their Ramond-Ramond partners, will give the Higgs branch of the field theory.) The five-dimensional version of this transition was discussed in [7], where it was seen that the field theory description of $A_{N-1}$ type as described in the previous section applies.

The $D_{N}$ theories are obtained when a birationally ruled surface over a $\mathbb{P}^{1}$ base collapses. The relevant light particles arise by wrapping a two-brane over various two-cycles. The quantization of their collective coordinates proceeds as in [7] and determines their quantum numbers. Wrapping the two-brane over the entire collapsing fiber we find vector multiplets in the adjoint representation of $S U(2)$. In the case analyzed in [7] the base was a Riemann surface of genus $g$ and hence there were also $g$ hypermultiplets in the adjoint representation which we do not have. Instead, we get hypermultiplets from the $2 \mathrm{~N}$ special $\mathbb{P}^{1}$ 's in our fiber. As in the $A_{k}$ cases, every such $\mathbb{P}^{1}$ leads to one hypermultiplet which is charged under the unbroken $U(1)$ with the minimal unit of charge. Therefore, every pair of $\mathbb{P}^{1}$ 's leads to a hypermultiplet which is an $S U(2)$ doublet. To summarize, the low energy spectrum is that of an $S U(2)$ gauge theory with $N$ hypermultiplets which are $S U(2)$ doublets. This is the spectrum of the $D_{N}$ field theories of the previous section.

When the surface $S$ collapses, there is an effective field theory description near $\phi=0$ (which labels the point of collapse in the Kähler moduli space). The gauge coupling will take the form $t(\phi)=t_{0}+c \phi$; since $c$ also multiplies the Chern-Simons term in the action, it can be calculated in terms of the intersection theory on $X$ [5, 6, 8 and it turns out to be $c=2 S \cdot S \cdot S$. (The factor of 2 is a normalization designed to match our field theory conventions.) More generally, the second derivative of the prepotential with respect to the field corresponding to $S$ takes the form $2 S \cdot S \cdot H$, where $H$ is an arbitrary divisor from the Kähler cone on $X$. Expanding $H$ in terms of a basis of $H^{1,1}(X)$ which contains $S$, we obtain an expression for the gauge coupling of the form

$$
\begin{aligned}
t(\phi) & =2 S \cdot S \cdot\left(\sum \alpha_{i} H_{i}+\phi S\right) \\
& =2 S \cdot S \cdot\left(\sum \alpha_{i} H_{i}\right)+c \phi=t_{0}+c \phi .
\end{aligned}
$$

Of course, any divisors $H_{i}$ with $S \cdot S \cdot H_{i}=0$ can be omitted from this expression.

In the simplest version of the $D_{N}$ theories - the ones with $k=1$ - there are only two independent elements in $H^{1,1}(X)$ which are not orthogonal to $S \cdot S$; we choose a basis for these consisting of $S$ and a divisor $H_{0}$ with $H_{0} \cdot S=\frac{1}{4} \mathcal{F}$, where $\mathcal{F}$ is a generic fiber of the ruling. Then the gauge coupling is given by

$$
t(\phi)=2 S \cdot S \cdot\left(t_{0} H_{0}+\phi S\right)=-2 K_{S} \cdot\left(\frac{t_{0}}{4} \mathcal{F}-\phi K_{S}\right)=t_{0}+(16-2 N) \phi .
$$


The factor of $1 / 4$ was included in the definition of $H_{0}$ to make the constant term match our conventions from section 2 .

At the other extreme, if $k=N+1$, we will find that all of the parameters in the field theory can be realized in the geometry. In addition to the divisors $H_{0}$ and $S$, we choose divisors $H_{i}$ on $X$ such that $H_{i} \cdot S=\frac{1}{2} \mathcal{F}-\Gamma_{i}$, where $\Gamma_{i}$ is one of the components of a reducible fiber. Note that the other component $\Gamma_{i}^{\prime}$ of that fiber then satisfies $\left(-H_{i}\right) \cdot S=\frac{1}{2} \mathcal{F}-\Gamma_{i}^{\prime}$, so the choice of component only affects the sign of $H_{i} .8$ If we write a general divisor in the form $t_{0} H_{0}+\sum_{i=1}^{N} m_{i} H_{i}+\phi S$ and assume it lies in the Kähler cone of $X$, then the gauge coupling is given by

$$
t(\phi)=2 S \cdot S \cdot\left(t_{0} H_{0}+\sum_{i=1}^{N} m_{i} H_{i}+\phi S\right)=t_{0}+(16-2 N) \phi,
$$

since $S \cdot S \cdot H_{i}=0$. The Kähler cone of $X$ is determined in part by the conditions

$$
\begin{aligned}
& 0<\left(t_{0} H_{0}+\sum_{i=1}^{N} m_{i} H_{i}+\phi S\right) \cdot \mathcal{F}=2 \phi \\
& 0<\left(t_{0} H_{0}+\sum_{i=1}^{N} m_{i} H_{i}+\phi S\right) \cdot \Gamma_{i}=\phi+m_{i} \\
& 0<\left(t_{0} H_{0}+\sum_{i=1}^{N} m_{i} H_{i}+\phi S\right) \cdot \Gamma_{i}^{\prime}=\phi-m_{i} .
\end{aligned}
$$

The curves $\Gamma_{i}$ or $\Gamma_{i}^{\prime}$ can now be flopped to produce other birational models of $X$. Pick disjoint subsets $A$ and $B$ from $\{1, \ldots, N\}$, and flop $\Gamma_{i}$ when $i \in A$ and $\Gamma_{i}^{\prime}$ when $i \in B$. Then the Kähler cone of the flopped model is determined in part by the conditions

$$
\begin{gathered}
0<2 \phi, \\
0<\phi+m_{i}, \text { for } i \notin A \\
0<-\phi-m_{i}, \text { for } i \in A \\
0<\phi-m_{i}, \text { for } i \notin B \\
0<-\phi+m_{i}, \text { for } i \in B .
\end{gathered}
$$

There is also a new expression for the gauge coupling valid in that cone:

$$
t(\phi)=t_{0}+(16-2 N+2 \#(A)+2 \#(B)) \phi+2 \sum_{i \in A} m_{i}-2 \sum_{i \in B} m_{i}
$$

8 However, as we learned in section 2, the sign is important; the one given here will be the correct choice once we get to the $E$ theories. 
(since $S \cdot S \cdot H_{i}$ decreases by 1 for $i \in A$ and increases by 1 for $i \in B$ when we move to the new cone). These formulas can all be put together into a single piecewise linear function

$$
t(\phi)=2 S \cdot S \cdot\left(t_{0} H_{0}+\sum_{i=1}^{N} m_{i} H_{i}+\phi S\right)=t_{0}+16 \phi-\sum_{i=1}^{N}\left|\phi+m_{i}\right|-\sum_{i=1}^{N}\left|\phi-m_{i}\right|
$$

valid throughout the union of all of the Kähler cones, and precisely reproducing eqn. (2.6). This firmly establishes our identification of not only the field theory, but also the parameters in it.

Now we have enough data to identify the interacting field theories $E_{N+1}$ in geometric terms. We start with the $D_{N}$ theory, realized geometrically as above with some value of $k$. Notice that the surface $S$ in this case is actually a blowup of $\mathbb{P}^{1} \times \mathbb{P}^{1}$ at $N$ points. We assume $N \leq 7$, and let $t_{0} \rightarrow 0$ and $\phi \rightarrow 0$, and well as $m_{i} \rightarrow 0$ for any parameters $m_{i}$ which are present. This means that we are approaching a part of the cone in which the entire surface $S$ collapses to a point, i.e., $X$ experiences the collapse of a del Pezzo surface. In other words, the del Pezzo contractions precisely realize the interacting five-dimensional field theories. (We have not yet established this in the cases of $E_{0}$ and $\widetilde{E}_{1}$, but we shall do so shortly.)

As in the $A_{N}$ and $D_{N}$ cases we can examine the BPS states which approach zero mass at the transitions. First, we could wrap various two-cycles with two-branes to find light particles. We could also wrap a five-brane over the entire collapsing surface $S$ to yield a string whose tension approaches zero. This string is electric-magnetic dual to the light particles. Such a spectrum in five dimensions was first observed in [7] for one of the del Pezzo contractions. Having both massless particles and tensionless strings at the transition points shows that they are not free field theories. We identify them with interacting field theories.

Before exploring the geometry of these del Pezzo contractions further, we pause to point out one of the striking consequences of this identification: the Higgs branches of generic extremal transitions are given by an $A, D$, or $E$ instanton moduli space. This prediction serves to unify a collection of disparate known facts about these transitions, which had not previously been seen as part of any pattern. Let us review what is known mathematically.

Given $S \subset X$ contracted by $X \rightarrow \bar{X}$, the mathematical question is whether $\bar{X}$ can be smoothed by varying its equations, and if so, what is the number of new parameters, i.e., the difference $\operatorname{dim} \operatorname{Def}(\bar{X})-\operatorname{dim} \operatorname{Def}(X)$ between the dimensions of the deformation spaces. (Note that this difference will coincide with the dimension of the Higgs branch in the field theory, although strictly speaking before making such a comparison we should 
construct the hypermultiplet moduli space in the string theory which requires the inclusion of Ramond-Ramond partners to the complex structure moduli.) The known facts about this mathematical question are as follows:

(1.) In the $A_{N-1}$ case, as pointed out above, an extremal transition exists whenever $N>k$ [26] and the number of new parameters is $N-k$ (as is seen by calculating $h^{2,1}$ [27,28]).

(2.) In the $D_{N}$ case, assuming for simplicity that $k=1,9$ an extremal transition exists whenever $N \geq 2$ [29]. The case $N=2$ is somewhat special, in that the deformation space for $\bar{X}$ is not smooth, but has two components of dimension one 30 [ as expected from the field theory, since $D_{2}$ is not simple). Our prediction for the number of new parameters is $2 N-k-2$ in general, and this is consistent with all known facts, including the examples worked out in 12$]$.

(3.) The $E_{N+1}$ cases are rather complicated, and one must consider different values of $N$ separately (cf. [31]); we again assume $k=1$ for simplicity.

(a.) For $\widetilde{E}_{1}$, and $E_{N+1}, 0 \leq N+1 \leq 3$, the singularity of $\bar{X}$ is toric and the deformation theory has been worked out by Altmann [32]; the result is that there is no smoothing for $E_{0}$ or $\widetilde{E}_{1}$ (this is a classic result of Schlessinger [33 in the $E_{0}$ case, which is a $\mathbb{Z}_{3}$ quotient singularity), a one-dimensional deformation space for the cases of $E_{1}$ and $E_{2}$, and a deformation space with two components, one of dimension one and one of dimension two, in the case of $E_{3}$. In addition, there are further, obstructed first-order deformations in precisely the $\widetilde{E}_{1}$ and $E_{2}$ cases - the ones with a $U(1)$ factor in their symmetry groups.

(b.) For $E_{4}$, the singularity of $\bar{X}$ is defined by a Pfaffian ideal and the theory of those can be used to study the deformations [34, 35]; the result is that a smoothing exists.

(c.) For $E_{N+1}, 5 \leq N+1 \leq 8$, the singular of $\bar{X}$ is a complete intersection and the deformation theory is relatively easy; smoothings exist in all cases.

For these del Pezzo contractions, it was observed in [21] that at least for $N+1 \geq 5$, the number of new parameters for this extremal transition should be $c_{N+1}-k$, where $c_{N+1}$ is the Coxeter number of the group $E_{N+1}$. In fact, this statement should continue to hold for all values of $N$, if properly interpreted: when the group $E_{N+1}$ is not simple, there must be two components whose dimensions are given by the Coxeter numbers (minus $k$ ) of the two factors in the group. (One should also include an obstructed deformation if there is a $U(1)$ factor.) This formula is compatible with all known calculations of dimensions of smoothing components. 11

9 This is the assumption " $X$ is $\mathbb{Q}$-factorial" which sometimes appears in the mathematics literature.

10 This was only shown in an example, but it probably holds in general.

11 We thank Mark Gross for discussions on this point. 
It is quite remarkable how this prediction about the Higgs branch unifies phenomena such as the two components or other obstructed deformations which appeared in sporadic locations (and looked in the past like counterexamples to any regularity of behavior). It also makes clear why the main theorems of [31] and [29] are so similar (as remarked in [29]).

As in the $D_{N}$ cases, when $k>1$ so that some of the field theory parameters are realized in the string theory, the $E_{N+1}$ theories will have a rich structure with various possible flops leading to new (Kähler) cones in which the gauge coupling takes a different form. This structure can be completely analyzed using the known combinatorial structure of the del Pezzo surfaces. In brief, the condition which must be satisfied in order to flop some collection of rational curves is that the del Pezzo surface can be blown down along those curves. The Weyl group of $E_{N+1}$ acts in a natural way 12 on $H^{1,1}(S)$, and permutes the various collections of curves which can be blown down. This can be used to write down the complete phase diagram for each case, and of course the gauge coupling can be directly calculated in every phase. This is essentially the same combinatorial data which we used in the field theory, so it is not surprising that the results turn out to be the same.

We will carry this out in the case of $N=1$, in order to complete our geometric identifications of the interacting field theories by including the $\widetilde{E}_{1}$ and $E_{0}$ cases.

We take a del Pezzo surface $S$ with $N=1$, embedded on a Calabi-Yau manifold $X$ with $k=3$. The surface $S$ can be regarded as a blowup of $\mathbb{P}^{2}$ at two points $P_{1}, P_{2}$; we let $\Gamma_{1}$ and $\Gamma_{2}$ be the exceptional divisors of the blowup. There is another exceptional divisor on $S$, a curve $\Gamma_{3}$ which is the proper transform of the line passing through $P_{1}$ and $P_{2}$.

We also introduce three divisors $H_{\ell}, H_{1}$, and $H_{2}$, which intersect $S$ in the classes of a line, and the two rulings. Specifically, $H_{1} \cdot S=\Gamma_{2}+\Gamma_{3}$ is in the same class as the proper transform of lines passing through $P_{1}$, and $H_{2} \cdot S=\Gamma_{1}+\Gamma_{3}$ is in the same class as the proper transform of lines passing through $P_{2}$. We write a general divisor on $X$ (modulo those orthogonal to $S \cdot S$ ) in the form

$$
a_{1} H_{1}+a_{2} H_{2}+a_{3} H_{\ell} .
$$

It is known [22] that on any del Pezzo surface, the walls of the Kähler cone are dual to the possible exceptional curves on the surface, which in our example are precisely $\Gamma_{1}$, $\Gamma_{2}$, and $\Gamma_{3}$. Thus, the Kähler cone of $X$ is defined by

$$
\begin{aligned}
& 0<\left(a_{1} H_{1}+a_{2} H_{2}+a_{3} H_{\ell}\right) \cdot \Gamma_{1}=a_{1} \\
& 0<\left(a_{1} H_{1}+a_{2} H_{2}+a_{3} H_{\ell}\right) \cdot \Gamma_{2}=a_{2} \\
& 0<\left(a_{1} H_{1}+a_{2} H_{2}+a_{3} H_{\ell}\right) \cdot \Gamma_{3}=a_{3} .
\end{aligned}
$$

12 This was known a long time ago [19,20], and provides an important clue to the $E_{N+1}$ symmetry in the physics [21]. 
We now consider the birational models of $X$ obtained by flopping these curves. We label the model by the type of surface $S$ has become on that model, so the original CalabiYau is labeled $X_{B l_{2} \mathbb{P}^{2}}$ (a two-point blowup of $\mathbb{P}^{2}$ ). We could flop $\Gamma_{3}$ to get a model $X_{\mathbb{F}_{0}}$ with $S=\mathbb{P}^{1} \times \mathbb{P}^{1}$. (We introduce the slightly simpler (standard) notation of $\mathbb{F}_{0}$ to denote $\mathbb{P}^{1} \times \mathbb{P}^{1}$ and $\mathbb{F}_{1}$ to denote $B l_{1} \mathbb{P}^{2}$; we will distinguish the blowups of $\mathbb{P}^{2}$ at $P_{1}$ and $P_{2}$ by labeling the latter as $\widetilde{\mathbb{F}}_{1}$.) On this model, $\Gamma_{1}$ and $\Gamma_{2}$ meet $H_{\ell}$, and the Kähler cone is defined by

$$
a_{1}+a_{3}>0, \quad a_{2}+a_{3}>0, \quad-a_{3}>0 .
$$

Or, we could flop $\Gamma_{1}$ to get a model $X_{\mathbb{F}_{1}}$ on which $\Gamma_{3}$ meets $H_{1}$ so that the Kähler cone is

$$
-a_{1}>0, \quad a_{2}>0, \quad a_{1}+a_{3}>0 .
$$

Similarly, if we flop $\Gamma_{2}$ then $\Gamma_{3}$ meets $H_{2}$ and we get a model $X_{\widetilde{\mathbb{F}}_{1}}$ whose Kähler cone is

$$
a_{1}>0, \quad-a_{2}>0, \quad a_{2}+a_{3}>0 .
$$

Finally, we could flop both $\Gamma_{1}$ and $\Gamma_{2}$ to get a model $X_{\mathbb{P}^{2}}$ with $\Gamma_{3}$ meeting both $H_{1}$ and $H_{2}$ so that the Kähler cone is

$$
-a_{1}>0, \quad-a_{2}>0, \quad a_{1}+a_{2}+a_{3}>0 .
$$

All five of these Kähler cones are illustrated in figure 2.

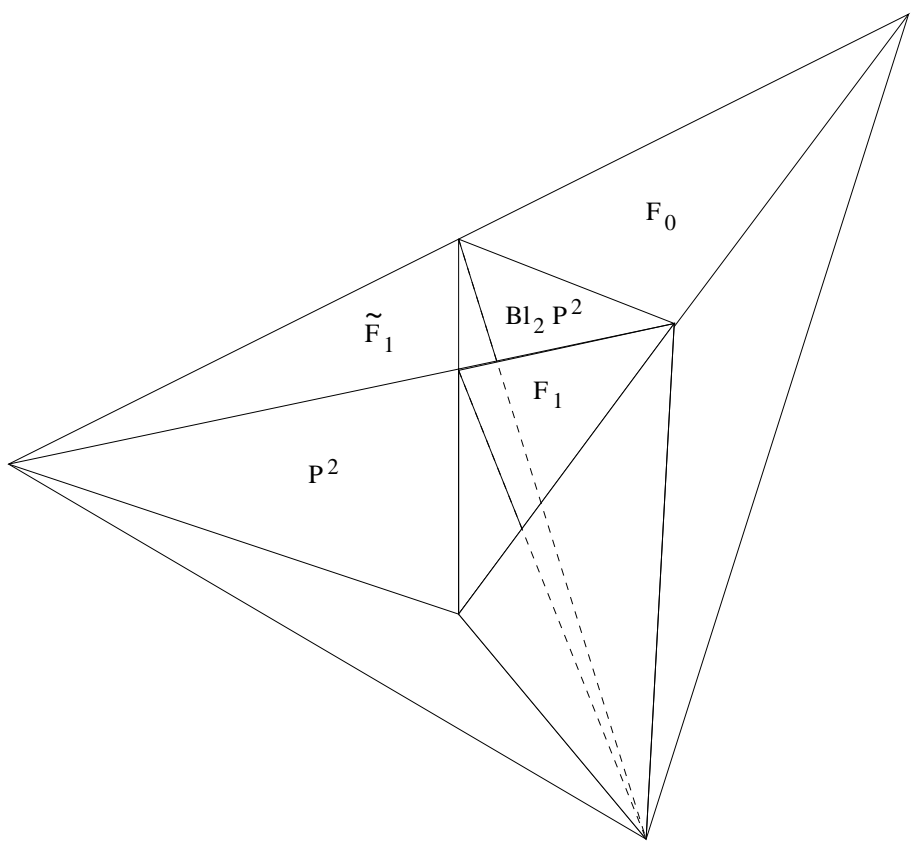

Figure 2. Phases for a del Pezzo contraction with $N=1$. 
We now ask what happens when we approach one of the boundary walls which does not correspond to a flop. We must either have a ruling along which the surface is contracted, or the surface must be a del Pezzo, contracted by its generator. The $\mathbb{F}_{0}$ case has two rulings $\Gamma_{1}$ and $\Gamma_{2}$, and the corresponding coefficients $a_{1}+a_{3}$ and $a_{2}+a_{3}$ when sent to zero produce $D_{0}$ theories. One sees an $E_{1}$ theory along the line of intersection of those boundary walls, where the entire $\mathbb{F}_{0}$ is contracted to a point.

Each of the $\mathbb{F}_{1}$ 's has a ruling (given by $\Gamma_{3}$ ), and contraction of these produces further $D_{0}$-type theories. To facilitate comparison with the field theory analysis, we label these particular theories as " $\widetilde{D}_{0}$ theories," although as in the field theory case the differences are minor. At the boundary between the contraction of one of the $\mathbb{F}_{1}$ 's and the contraction of the corresponding ruling on $\mathbb{F}_{0}$ (as detected by the coefficients being the same), we find a $D_{1}$ theory - the entire surface $B l_{2} \mathbb{P}^{2}$ is contracted along the ruling in this case.

Finally, along the boundary of the $\mathbb{P}^{2}$ cone at which $a_{1}+a_{2}+a_{3}$ goes to zero, we get an $E_{0}$ theory. Between this $E_{0}$ theory and the neighboring $\widetilde{D}_{0}$ theory one finds an $\widetilde{E}_{1}$ theory, in which the $\mathbb{F}_{1}$ or $\widetilde{\mathbb{F}}_{1}$ has been contracted to a point. Furthermore, at the vertex of this part of the cone, all three classes from $B l_{2} \mathbb{P}^{2}$ have been contracted and we get a theory of type $E_{2}$.

These boundary behaviors are illustrated in figure 3, where to simplify the drawing we show only a slice of the cone from figure 2 (so the $E_{2}$ point is omitted entirely). To compare with the field theory illustrated in figure 1, one should imagine taking the boundary of the cone of figure 2 and flattening it onto the plane. The labels along the edges in figure 3 would then label the regions in the boundary of the cone, which are easily seen to precisely correspond to figure 1 .

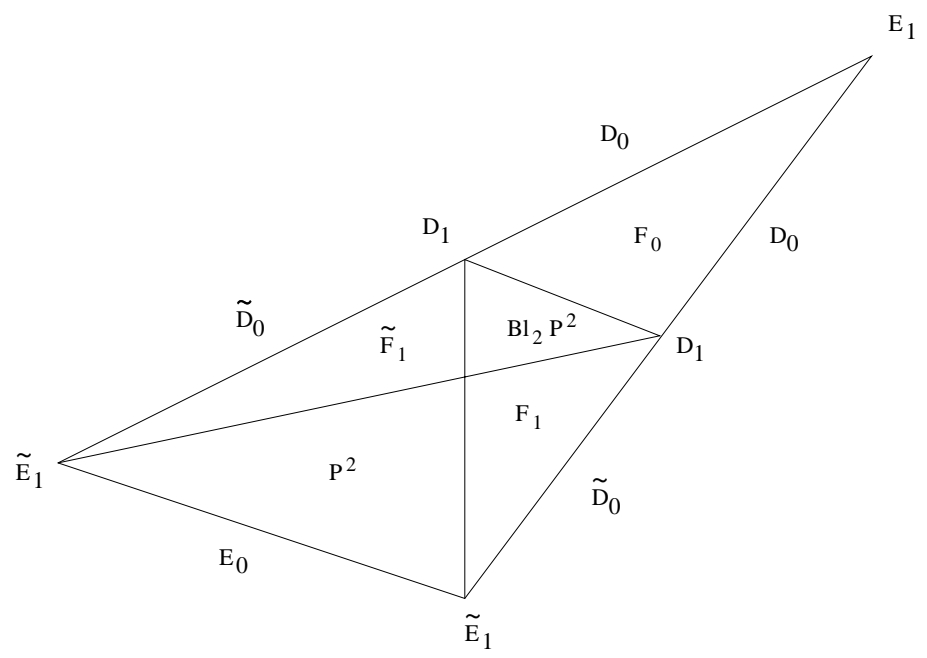

Figure 3. Cross-section of the phase diagram, showing boundary behavior. 
In fact, it is this detailed correspondence which allows us to identify with confidence the $E_{1}$ and $\widetilde{E}_{1}$ field theories with the $\mathbb{F}_{0}=\mathbb{P}^{1} \times \mathbb{P}^{1}$ and $\mathbb{F}_{1}=B l_{P} \mathbb{P}^{2}$ del Pezzo surfaces, respectively. Further evidence that these identifications are correct is provided by the perfect correspondence between the mathematical deformation theory of $\bar{X}$, and the structure of the Higgs branch of the field theory (which is different in the two cases).

\section{Acknowledgments}

The work of D.R.M. was supported in part by NSF grant DMS-9401447, and that of N.S. was supported in part by DOE grant DE-FG02-96ER40559. We thank M. Douglas, B. Greene, M. Gross, S. Shenker, C. Vafa, N. Warner, and E. Witten for helpful discussions.

\section{Appendix A. Non-generic primitive contractions}

The list we have given in section 3 of possible primitive extremal contractions from a Calabi-Yau threefold is not complete. (A "primitive" contraction is one which occurs at codimension one in the Kähler cone.)

In the case of curves collapsing to points, in principle any terminal singularity could occur. However, this should not happen at generic complex moduli, in light of some results of Namikawa [36]. Although he did not state things precisely in this way, the proof of Theorem (2.5) of [36] suggests that after a primitive extremal contraction $X \rightarrow \bar{X}$ where $\bar{X}$ has terminal singularities, one should be able to deform away the non-ordinary double points on $\bar{X}$ while keeping the ordinary double points. The implication would be that at generic complex moduli of $X$ only ordinary double points should appear in such contractions. This question deserves further study.

In the case of a divisor collapsing to a point, the original classification goes back to Reid's first paper on canonical singularities [37], in which it was shown that the divisor must be a "generalized del Pezzo surface." Such surfaces include nonsingular del Pezzo surfaces (blowups of $\mathbb{P}^{2}$ or $\mathbb{P}^{1} \times \mathbb{P}^{1}$ with ample anti-canonical bundle), del Pezzo surfaces with rational double points (which will deform to the nonsingular ones), nonnormal del Pezzo surfaces, and cones over elliptic normal curves. As Mark Gross has pointed out [31], the latter can only occur as exceptional divisors of primitive contractions from a nonsingular Calabi-Yau if the degree is 1, 2, or 3, and those cones will deform to del Pezzo surfaces of types $E_{8}, E_{7}$ and $E_{6}$, respectively.

The troublesome case is the nonnormal del Pezzo surfaces [38]. They include analogous cones over nodal or cuspidal rational curves, and Gross's argument also limits those to the 
three low-degree cases (which deform to $E_{8}, E_{7}$, and $E_{6}$ ). Of the remaining possibilities, Gross shows that only one - a nonnormal del Pezzo of degree 7, corresponding to some sort of degenerate form of $E_{2}$ - could occur. We believe that this will not occur at generic complex structure, but have no concrete evidence to support this belief.

Finally, we come to the most technically difficult case of a divisor $S$ collapsing to a curve, analyzed in [29]. When the curve has genus at least one, there is a deformation of $X$ under which the divisor ceases to be effective [18,29]; thus, this case does not occur when the complex structure is generic. (This is also expected on physical grounds [11], since the field theory description consists of $S U(2)$ with charged matter in $g \geq 1$ adjoints, and possibly some fundamentals as well.) In the case of contraction to a curve of genus zero, there is the possibility that curves being contracted are generically two $\mathbb{P}^{1}$ 's which meet; according to [29], for generic complex structure this can only happen in the case of $S^{3}=7$, i.e., some kind of degenerate form of $D_{1}$. We believe that this case too will not occur at generic complex structure, but have no concrete evidence to support this belief either. (Note that 29], Example 1.5, shows that such surfaces can indeed occur on Calabi-Yau threefolds.) 


\section{References}

[1] For a nice review see, S. Chaudhuri, C. Johnson, and J. Polchinski, "Notes on DBranes," hep-th/9602052.

[2] N. Seiberg, "IR Dynamics on Branes and Space-Time Geometry," hep-th/9606017.

[3] N. Seiberg, "Five Dimensional SUSY Field Theories, Non-trivial Fixed Points, and String Dynamics," hep-th/9608111.

[4] J. Polchinski and E. Witten, "Evidence for Heterotic - Type I Duality," Nucl. Phys. B460 (1996) 525-548, hep-th/9510169.

[5] A. C. Cadavid, A. Ceresole, R. D'Auria, and S. Ferrara, "Eleven-Dimensional Supergravity Compactified on a Calabi-Yau Threefold," Phys. Lett. B357 (1995) 76-80, hep-th/9506144.

[6] S. Ferrara, R.R. Khuri, and R. Minasian, "M-Theory on a Calabi-Yau Manifold," Phys.Lett. B375 (1996) 81-88 hep-th/9602102.

[7] E. Witten, "Phase Transitions in M-Theory and F-Theory," hep-th/9603150.

[8] S. Ferrara, R. Minasian, and A. Sagnotti, "Low Energy Analysis of $M$ and $F$ Theories on Calabi-Yau Threefolds", hep-th/9604097.

[9] A. Strominger, "Massless Black Holes and Conifolds in String Theory," Nucl. Phys. B451 (1995) 97-109, hep-th/9504090.

[10] B. R. Greene, D. R. Morrison, and A. Strominger, "Black Hole Condensation and the Unification of String Vacua," Nucl. Phys. B451 (1995) 109-120, hep-th/9504145.

[11] S. Katz, D. R. Morrison, and M. R. Plesser, "Enhanced Gauge Symmetry in Type II String Theory," hep-th/9601108.

[12] P. Berglund, S. Katz, A. Klemm, and P. Mayr, "New Higgs Transitions Between Dual $\mathrm{N}=2$ String Models," hep-th/9605154.

[13] C. Vafa, Private communication.

[14] M. Douglas, S. Katz, and C. Vafa, to appear.

[15] N. Seiberg, "Naturalness Versus Supersymmetric Non-renormalization Theorems," Phys. Lett. B318 (1993) 469-475, hep-ph/9309335.

[16] P. C. Argyres, M. R. Plesser, and N. Seiberg, "The Moduli Space of Vacua of $N=2$ SUSY QCD and Duality in $N=1$ SUSY QCD," Nucl. Phys. B471 (1996) 159-194, hep-th/9603042.

[17] Y. Hayakawa, "Degeneration of Calabi-Yau Manifold with Weil-Petersson Metric," alg-geom/9507016.

[18] P. M. H. Wilson, "The Kähler Cone on Calabi-Yau Threefolds," Invent. Math. 107 (1992) 561-583; Erratum, ibid. 114 (1993) 231-233.

[19] M. Demazure, "Surfaces de del Pezzo, II, III, IV, V," Séminaire sur les Singularités des Surfaces, Lecture Notes in Math. vol. 777, Springer-Verlag, 1980, pp. 21-69. 
[20] Yu. I. Manin, Cubic Forms: Algebra, Geometry, Arithmetic, 2nd ed., North-Holland, Amsterdam, New York, 1986, Chapter IV.

[21] D. R. Morrison and C. Vafa, "Compactifications of F-Theory on Calabi-Yau Threefolds (II)," Nucl. Phys. B476 (1996) 437-469, hep-th/9603161.

[22] S. Mori, "Threefolds Whose Canonical Bundles are Not Numerically Effective," Annals of Math. (2) 116 (1982) 133-176.

[23] D. R. Morrison, "Through the Looking Glass," to appear.

[24] K. Becker, M. Becker, and A. Strominger, "Fivebranes, Membranes and Non-Perturbative String Theory," Nucl. Phys. B456 (1995) 130-152, hep-th/9507158.

[25] B. R. Greene, D. R. Morrison, and C. Vafa, "A Geometric Realization of Confinement," hep-th/9608039.

[26] R. Friedman, "Simultaneous Resolution of Threefold Double Points," Math. Ann. 274 (1986) 671-689.

[27] H. Clemens, "Double Solids," Adv. Math. 47 (1983) 107-230.

[28] C. Schoen, "On Fiber Products of Rational Elliptic Surfaces with Section," Math. Zeit. 197 (1988) 177-199.

[29] M. Gross, "Primitive Calabi-Yau Threefolds," alg-geom/9512002.

[30] M. Gross, "The Deformation Space of Calabi-Yau n-Folds Can Be Obstructed," Mirror Symmetry II (B. Greene and S.-T. Yau, eds.), to appear, llg-geom/9402014.

[31] M. Gross, "Deforming Calabi-Yau Threefolds," alg-geom/9506022.

[32] K. Altmann, "The Versal Deformation of an Isolated Toric Gorenstein Singularity," alg-geom/9403004.

[33] M. Schlessinger, "Rigidity of Quotient Singularities," Invent. Math. 14 (1971) 17-26.

[34] D. Buchsbaum and D. Eisenbud, "Algebra Structures for Finite Free Resolutions and Some Structure Theorems for Ideals of Codimension 3," Amer. J. Math. 99 (1977) $447-485$.

[35] H. Kleppe and D. Laksov, "The Algebraic Structure and Deformation of Pfaffian Schemes," J. Algebra 64 (1980) 167-189.

[36] Yo. Namikawa, "Stratified Local Moduli of Calabi-Yau 3-Folds," preprint, 1995.

[37] M. Reid, "Canonical 3-Folds," Journées de Géométrie Algébriqe d'Angers (A. Beauville, ed.), Sitjhoff \& Noordhoof, 1980, pp. 273-310.

[38] M. Reid, "Nonnormal del Pezzo Surfaces," Publ. Res. Inst. Math. Sci. 30 (1994) 695727, alg-geom/9404002. 